\title{
Effectiveness of Acromioclavicular Joint Mobilization and Physical Therapy vs Physical Therapy Alone in Patients with Frozen Shoulder: A Randomized Clinical Trial
}

\section{Mohammad Rahbar}

Tabriz University of Medical Sciences

\section{Sepideh Ranjbar Kiyakalayeh}

Tabriz University of Medical Sciences

\section{Bina Eftekharsadat}

Tabriz University of Medical Sciences

\section{Behzad Izadseresht}

Tabriz University of Medical Sciences

Neda Dolatkhah ( $\square$ neda_dolatkhah@yahoo.com )

Tabriz University of Medical Sciences

\section{Research Article}

Keywords: Acromioclavicular joint mobilization, Physical therapy, Visual analogue scale (VAS), Shoulder pain and disability index (SPADI)

Posted Date: June 18th, 2021

DOI: https://doi.org/10.21203/rs.3.rs-548390/v1

License: (c) (i) This work is licensed under a Creative Commons Attribution 4.0 International License.

Read Full License

Version of Record: A version of this preprint was published at Clinical Rehabilitation on December 29th, 2021. See the published version at https://doi.org/10.1177/02692155211070451. 


\section{Abstract}

Background: Frozen shoulder (FS) is a prevalent musculoskeletal condition characterized by an often prolonged pain, disability and limited active and passive range of motion (ROM), however its management remains challenging yet. The objective of this trial was to compare the efficacy of acromioclavicular joint mobilization and physical therapy versus physical therapy in treatment of FS.

Methods: In this single-blind randomized clinical trial, patients with diagnosis of FS were randomly allocated into mobilization + physical therapy $(n=28)$ as experiment group, and physical therapy $(n=28)$ as control group in two outpatient clinics of Tabriz University of Medical Sciences, Iran. The primary outcomes were self-reported shoulder pain-related disability measured by the Shoulder Pain and Disability Index (SPADI) questionnaire and goniometric assessment of shoulder ROM. The secondary outcome was the Visual Analogue Scale (VAS). Measures were performed at the baseline, immediately and one month after beginning the treatments.

Results: Totally 51 patients with $25.73 \pm 6.88$ years old of age completed the study and their data were analyzed. VAS, SPADI, pain and disability improved more significantly in experiment group compared to control group immediately [-4.63 (-5.58- -3.67) vs. $-2.22(-2.96--1.47), p<0.001 ;-23.08(-28.63--17.53)$ vs. $-13.04(-17.93--8.16), p=0.008 ;-26.00(-31.71--20.29)$ vs. $-16.35(-23.39--9.31), p=0.034$ and -21.25 $(-29.11--13.39)$ vs. $-10.98(-17.53-4.43), p=0.042$, respectively] and one month after beginning of treatment $[-5.58(-6.45--4.72)$ vs. $-3.61(-4.60--2.62), p<0.001 ;-33.43(-40.85--26.01)$ vs. $-20.03(-26.00-$ $-14.07), p=0.001 ;-42.83(-49.09--36.57)$ vs. -25.57 (-33.92- -17.21$), p<0.001$ and $-27.55(-36.19--15.94)$ vs. -16.58 (-24.48- -8.67), $p=0.041$, respectively]. Active abduction ROM was also improved more significantly immediately after treatment in experiment group compared to control group [25.83 (11.45-40.13) vs. 10.17 (1.02-19.15), $p=0.025]$, however there were no significant differences between two groups concerning other measured ROMs immediately and one month after treatment (all $p>0.05$ ).

Conclusions: Acromioclavicular mobilization along with conventional physical therapy was more efficient in decreasing pain and disability and improving active abduction ROM compared to physical therapy in patients with FS. These findings would suggest a new therapeutic method for shoulder disorders with pain and disability.

\section{Trial registration}

www.irct.ir, IRCT20100605004104N7, registered 06.01.2019 (https://www.irct.ir/trial/35900)

\section{Introduction}

Frozen shoulder (FS) also recognized as adhesive capsulitis, is one of the most common clinical exhibitions in physical therapy practice [1]. It is a condition described by substantial shoulder stiffness, loss of active and passive range of motion (ROM) of the glenohumeral joint along with severe pain that typically intensifies at night [2]. FS is supposed to have a prevalence of $3-5 \%$ in the general population 
and up to $20 \%$ in persons with diabetes mellitus and it happens more frequently in women; the nondominant side is more probable to develop FS [3-5]. Conventionally, FS has been considered as a selflimiting disorder. Nonetheless, as it became apparent that the disability due to this condition lasts for years without suitable management, several treatment techniques have been suggested [6, 7]. Shaffer et al [8] reported that half of FS patients had symptoms at a mean of seven years from the beginning of the disorder, while only $11 \%$ described functional disabilities. This dispossesses the patients of the routine life and work-related and entertaining activities.

Prevalent conventional treatments comprise oral medication, physical therapy, exercise, corticosteroid injection, and hydrodilatation [9]. Several physical therapies and home-based trainings can be applied as a first-line treatment approach for FS [10]. Non-steroidal anti-inflammatory drugs (NSAIDs) along with physiotherapy were established to be more efficient in comparison with using NSAIDs alone [11]. Likewise, several investigations on intra-articular corticosteroid injections applied along with physical therapy caused improved consequences in comparison with corticosteroid injections alone [12,13]. Kelley et al [14] has published recent clinical practice recommendations and guidelines for the treatment of patients with FS. The treatments were included corticosteroid injections, patient education, physical therapy modalities, joint mobilizations, translational mobilizations, manipulations, and exercises. There are some preceding investigations for the usage of joint mobilization correlated to the rotation loss of the shoulder joint among patients with FS [15-17]. There are some details to propose that mobilization methods may be efficient in decreasing pain and disability in patients with FS. Mobilization methods are frequently applied to improve ROM. Several recommendations for mobilization procedures are accessible, but it is still controversy what the ideal direction of force and movement application should be to reestablish joint mobilization in patients with FS [18].

FS has four phases: the pre-adhesive stage, the acute adhesive or "freezing" stage, the fibrotic or "frozen" stage, and the "thawing" phase [11]. From the clinical point of view, patients commonly undergo physical therapy in stage two and three. In these phases, pain is somewhat decreased, nonetheless there are adhesions in the joint capsule in addition to limitations in the joint movements (both active and passive) [19].

The acromioclavicular joint (ACJ) displays the connection between the scapula and the clavicle, which is accountable for the coordinated dynamic of the shoulder girdle [20]. It gives further range of rotation on the thorax for the scapula, which lets the scapula to redact outside of its primary plane (posterior bending and internal rotation) to conform the altering the thorax shape as arm motion happens [21,22]. Current biomechanical investigations confirmed the ACJ's important role in total motion of the shoulder [21, 2325].

Several studies have been done to explore the effects of mobilization therapy in the management of FS [26-29]. Nevertheless, as to our knowledge no studies have been done to look at whether specific ACJ mobilization will provide any additional beneficial properties. So, the objective of this trial is to evaluate 
the efficacy of ACJ mobilization techniques in the re-establishment of joint mobility and decrease pain and disability in patients with FS.

\section{Methods And Materials}

\section{Design and setting}

This trial was a single-blind parallel-group randomized clinical trial in which patients with FS were recruited from physical medicine and rehabilitation outpatient clinics (Imam Reza and Shohada) belong to Tabriz University of medical sciences, Iran between February 2019 and January 2020. The investigation has been done according to the principles of the Declaration of Helsinki and Good Clinical Practice. Patients were provided written informed consent before any measurement was done. The CONSORT guidelines were conformed, and the CONSORT flowchart was prepared to display the current of the patients at every stage of the trial.

\section{Participants}

The inclusion criteria were: 1) a diagnosis of unilateral primary or idiopathic FS [30], 2) pain and restricted movement present for more than one month [31] , 3) normal shoulder x-rays findings, 4) normal neurologic examination, and 5) 18 to 60 years of age. Patients with neurological disorders, such as in stroke, as well as those with uncontrolled diabetes, rheumatic disease in the shoulder, malignant tumors, a history of the fracture or subluxation in the affected shoulder, history of surgery to the injured shoulder, recent drug or alcohol usage or addiction, history of corticosteroid injection in glenohumeral joint, glenohumeral joint instability or radiating pain from a cervical severe radiculopathy were excluded. Patients referred to outpatient clinics were recruited by a trained physiatrist via non-random convenience sampling method.

The demographic and clinical characteristics of the patients were measured at the baseline.

\section{Randomization procedures}

Patients were randomly allocated, in a 1:1 ratio, to receive one of the subsequent trial treatments: 1 ) Group 1: The ACJ mobilization + standard physical therapy + exercises and 2) Group 2: Standard physical therapy+ exercises. Concealed allocation was directed by means of computer-generated random numbers generated by a person not included with enrolling patients. The randomized allocation, enrolling patients and assigning them to treatments, was performed by a trained physiotherapist at the outpatient clinic. Patients were requested not to discuss the specific treatment received with the examiner researcher. Research coordinators who evaluated patient end points and data analyst were blinded to the allocated intervention groups through the trial and while executing analyses; however, it was not probable to blind participants or treating therapists.

\section{Treatment Programs}


1) Group 1: ACJ mobilization + standard physical therapy + exercises

ACJ mobilization was performed in up to six 30-minute individual sessions within a 3-week period by a single trained therapist. The treatment techniques were anterior to posterior passive accessory glides of the distal end of the clavicle categorized from Grade I-IV [32].The patient is placed in the supine position and the upper limb is placed in a physiological position with the patient's arm clinging to the body and the hand on the abdomen, which causes the capsule to stretch less and the technique to be less painful. The therapist placed the tips of his both thumbs on the anterior surface of the clavicle adjacent to the ACJ and spread his other fingers out for stability and his forearm was situated in line with the posterior movement at the ACJ. Grade I means an intensity of slight amplitude that is used at the start of the ACJ joint ROM, with no pressure on connective tissue. Grade II means an intensity of a little larger amplitude used from the start of the joint ROM to the middle. Grade I and II are suitable for relieving pain. Grade III is used at a large amplitude from the middle of the joint ROM to the start of the constraint. Grade IV is used against the tissue resistance at a slight amplitude to the limited part of the joint. Grade III and IV are applied for stimulating a stretching to relieve joint stiffness in a shorter tissue [33-35].

2) Group 2: Standard physical therapy + exercises

Standard physical therapy was performed in 10 sessions (3 sessions per week). Physical therapy treatments included:

Hot pack (HP) as a surface heat modality on the upper trapezius muscle for 20 minutes at a temperature of $74.5^{\circ} \mathrm{C}$ to reduce muscle spasm

$1 \mathrm{MHz}$ ultrasound at an intensity of 1,5-1,25 W/cm2 for $5 \mathrm{~min}$ for shoulder joint capsule and rotator cuff muscle tendon [36].

TENS (Transcutaneous Electrical Nerve Stimulation) in the conventional mode for 25 minutes (lowintensity, small pulse width: $50-200 \mu$ s with a frequency of $50-100 \mathrm{~Hz}$ ) [37].

In both groups, shoulder exercises were performed at home for 15 minutes per time (10 repetitions of each), twice a day with direction by the physiotherapist once per week. These exercises included active ROM exercise (such as pendulum stretch (passively), placing one hand on the back of the neck and one hand on the back of the waist and then vice versa, active range of motion of the scapula, elbow circle, finger walk on the wall), towel stretch behind the back to improve internal and external rotations, stretching anterior capsule whereas standing next to the door and walking pass the door, cross body stretch for the posterior capsule, stretch arm above the head with the assistance of the healthy arm in lying or standing position and isometric shoulder internal and external rotation proceeded into active resistive to the extent that the patient's pain allows. Patients were instructed about how to perform the exercises in a pain-free manner so as to maximize normal muscle function and movement pattern [38] and provided as a pamphlet comprising the image and the description for all the exercises to 
confirm compliance. Furthermore, patients were instructed to avoid painful activities including the shoulder and were counseled how to apply pain-free approaches to do daily activities.

\section{Primary and secondary outcome measures}

The primary outcomes were self-reported shoulder pain-related disability measured by the Shoulder Pain and Disability Index (SPADI) questionnaire [39] and goniometric assessment of active shoulder ROM [33, 40].

SPADI was established to quantity present shoulder pain and disability in an outpatient setting. The SPADI comprises 13 pieces that evaluate two areas; a 5-piece subscale that measures pain and an 8piece subscale that measures disability [41], each element is recorded on a visual analogue scale (VAS). The patient was taught to place a spot on the VAS for each piece that best signified their experience of the shoulder condition over the last week [42]. Each subscale is summed and converted to a total out of 100. A mean is obtained from the two subscales to give a total score out of 100 , greater score representing higher disability [20]. We applied the Persian version of SPADI with confirmed validity and reliability in patients with shoulder conditions in research setting and clinical practice [43].

A metal goniometer was used to measure the ROM of the shoulder joint actively and passively in flexion, abduction and internal and external rotation movements. The ROM was measured in a sitting position while holding the patient's scapula with a stable hand [44].

The Shoulder hand behind back (HBB) ROM method was also applied for measuring active shoulder internal rotation with established validity in subjects with shoulder pain of mechanical origin. HBB ROM was defined by determining the interval in centimeters between the T1 spinous process and the radial styloid process and compared to the healthy side, the greater the difference between the two sides, the worse the ROM. HBB ROM is precise, has respectable inter- and intra-rater reliability, and provides normal values for between-limb ROM variability [20,45].

The secondary outcome was the Visual Analogue Scale (VAS). VAS was applied to measure the pain intensity. VAS is a fast (statistically quantifiable and reproducible) measurement of pain intensity. Patients were asked to place a vertical mark along a 10-cm-long horizontal line indicating the severity of their pain $(0=$ no pain, $10=$ the worst pain) [21].

\section{Statistical analysis}

Using G-POWER 3.1.9.2 (Faul, Erdfelder, Lang, \& Buchner, 2007) software and based on the Çelik et al study [25] with $80 \%$ power, an alpha level of 0.05 and Effect size $=0.44$, the total sample size was calculated to be 50 ( 25 in each group). An allowance was made for a $10 \%$ dropout rate, increasing the sample size to 56 patients (28 per group).

All data achieved from the trial were analyzed by the SPSS 17.0 software (IBM SPSS Statistics for Windows, Version 17.0. Armonk, NY: IBM Corp) with the level of significance considered at $p<0.05$. The 
normal distribution of different variables was evaluated using the Shapiro-Wilk test. The Mann-Whitney Utest and the $\chi 2$ test were applied for intergroup comparisons of baseline data. Mixed ANOVA test (time [within subject] * group [between subjects]) in conjunction with the sidak posthoc as adjustment method were used to estimate within group alterations and between group differences. We termed effect size as Cohen's $d$ for outcome measures. We measured the effect sized of $0.2,0.5$ and 0.8 as small, medium and large, respectively [43].

\section{Results}

The flow chart of participants is presented in Fig. 1. Fifty-six patients were randomly assigned to the experimental and control groups, from which three patients in the experimental group and two in control group excluded from the study due to not completing the treatment period and not referring for follow-up. The general characteristics of the patients are shown in Tables 1 and 2 . Study groups had comparable demographic and anthropometric characteristics $(P>0.05)$ and baseline outcome measurements $(P>$ 0.05). 
Table 1

Demographic characteristics of patients participating in the study by trial arm

\begin{tabular}{|c|c|c|c|c|}
\hline \multicolumn{2}{|l|}{ Variables } & $\begin{array}{l}\text { Mobilization group } \\
(n=28)\end{array}$ & $\begin{array}{l}\text { Control group } \\
(n=28)\end{array}$ & p-value \\
\hline \multicolumn{2}{|l|}{ Age (years) } & $54.50 \pm 6.39$ & $54.91 \pm 6.11$ & $0.822^{\star}$ \\
\hline \multirow[t]{2}{*}{ Sex } & Male & $11(39.3 \%)$ & $10(35.7 \%)$ & \multirow[t]{2}{*}{$0.846 * \star$} \\
\hline & Female & $17(60.7 \%)$ & $18(64.3 \%)$ & \\
\hline \multicolumn{2}{|c|}{ Disease duration (months) } & $7.58 \pm 5.22$ & $8.21 \pm 10.30$ & $0.755^{\star}$ \\
\hline \multirow[t]{4}{*}{ Occupation } & Workless & $3(10.8 \%)$ & $2(7.2 \%)$ & \multirow[t]{4}{*}{$0.901^{* *}$} \\
\hline & Housewife & $16(57.1 \%)$ & $15(53.6 \%)$ & \\
\hline & Employed & $4(14.3 \%)$ & $5(17.8 \%)$ & \\
\hline & Retired & $5(17.8 \%)$ & $6(21.4 \%)$ & \\
\hline \multirow[t]{3}{*}{ Affected side } & Illiterate/ Primary school & $4(18.2 \%)$ & $6(25.0 \%)$ & \multirow[t]{3}{*}{$0.604^{\star *}$} \\
\hline & High school & $14(63.6 \%)$ & $15(62.5 \%)$ & \\
\hline & Higher education & $4(18.2 \%)$ & $3(12.5 \%)$ & \\
\hline \multicolumn{2}{|l|}{ VAS (cm) } & $7.71 \pm 1.89$ & $7.83 \pm 1.47$ & $0.814^{\star}$ \\
\hline \multicolumn{2}{|l|}{ SPADI (\%) } & $64.33 \pm 19.21$ & $66.86 \pm 13.61$ & $0.606^{*}$ \\
\hline \multicolumn{2}{|c|}{ SPADI: Pain (\%) } & $67.08 \pm 20.26$ & $69.83 \pm 20.88$ & $0.650^{\star}$ \\
\hline \multicolumn{2}{|c|}{ SPADI: Disability (\%) } & $62.60 \pm 22.53$ & $65.00 \pm 19.78$ & $0.701^{\star}$ \\
\hline
\end{tabular}


Table 2

Mean ROM of patients participating in the study by trial arm.

\begin{tabular}{|c|c|c|c|}
\hline Variables & $\begin{array}{l}\text { Mobilization group } \\
(n=28)\end{array}$ & $\begin{array}{l}\text { Control group } \\
(n=28)\end{array}$ & p-value \\
\hline Active Flexion ROM $\left(^{\circ}\right)$ & $104.42 \pm 11.68$ & $104.26 \pm 17.64$ & $0.972 *$ \\
\hline Active Abduction ROM $\left(^{\circ}\right)$ & $89.88 \pm 9.93$ & $88.52 \pm 14.31$ & $0.707 *$ \\
\hline Active Internal Rotation ROM $\left(^{\circ}\right)$ & $24.04 \pm 6.57$ & $25.57 \pm 9.88$ & $0.535^{*}$ \\
\hline Active External Rotation ROM $\left(^{\circ}\right)$ & $52.17 \pm 16.30$ & $53.65 \pm 15.56$ & $0.794 *$ \\
\hline Passive Flexion ROM $\left(^{\circ}\right)$ & $110.29 \pm 12.69$ & $109.57 \pm 17.01$ & $0.869 *$ \\
\hline Passive Abduction ROM $\left(^{\circ}\right)$ & $92.87 \pm 11.28$ & $91.83 \pm 13.70$ & $0.775^{*}$ \\
\hline Passive Internal Rotation ROM $\left(^{\circ}\right)$ & $25.42 \pm 7.90$ & $26.39 \pm 11.04$ & $0.729 *$ \\
\hline Passive External Rotation ROM ( $\left.{ }^{\circ}\right)$ & $52.17 \pm 16.30$ & $53.65 \pm 15.56$ & $0.751^{*}$ \\
\hline HBB ROM $(\mathrm{cm})$ & $-17.05 \pm 9.22$ & $-17.05 \pm 9.22$ & $0.917 *$ \\
\hline
\end{tabular}

Substantial significant interaction was perceived between time points (before, immediately after intervention, one month after intervention) meaning as the within-group factor and group (mobilization + standard physical therapy + exercises vs. standard physical therapy + exercises) as the between-group factor concerning the study outcomes (VAS: $p$-value < 0.05; SPADI: $p$-value < 0.05; pain: $p$-value $<0.01$; disability: $p$-value $<0.01)$. Base on the interpretation of the Cohen's $d$ values, the findings referred to large effect size for study outcomes $(d=2.58, d=2.03, d=3.39$ and $d=2.43$ for VAS, SPADI, pain and disability, respectively). So we measured the variance between study groups at each level of time factor.

Table 3 shows he results on the analysis of clinical outcomes. A significant improvement in all studied variables in both groups was observed immediately after the end of treatment, which remains stable for up to one month after the intervention $(p<0.05)$. However, decreases in VAS and SPADI scores (total and pain and disability subscales) were significantly higher in mobilization group than the control group immediately after $(p<0.001, p=0.008, p=0.034$ and $p=0.042$ respectively $)$ and also one month after the intervention ( $p<0.001, p=0.001, p<0.001$ and $p=0.041$ respectively). 
Table 3

Outcome measurements among groups

\begin{tabular}{|c|c|c|c|c|c|c|}
\hline \multirow[t]{3}{*}{ Clinical value } & & \multicolumn{2}{|c|}{$\begin{array}{l}\text { Mobilization group } \\
(\mathrm{n}=25)\end{array}$} & \multicolumn{2}{|c|}{$\begin{array}{l}\text { Control group } \\
(n=26)\end{array}$} & \multirow[t]{3}{*}{$\begin{array}{l}\mathrm{P} \\
\text { Value }\end{array}$} \\
\hline & & \multirow{2}{*}{$\begin{array}{l}\text { Mean } \\
\pm \text { SD }\end{array}$} & MC & \multirow{2}{*}{$\begin{array}{l}\text { Mean } \\
\pm S D\end{array}$} & MC & \\
\hline & & & P Value * & & P Value * & \\
\hline \multirow[t]{5}{*}{ VAS } & Before & $\begin{array}{l}7.71 \pm \\
1.89\end{array}$ & Reference & $\begin{array}{l}7.83 \pm \\
1.47\end{array}$ & Reference & \\
\hline & \multirow[t]{2}{*}{$\begin{array}{l}\text { Immediately after } \\
\text { the intervention }\end{array}$} & \multirow[t]{2}{*}{$\begin{array}{l}3.08 \pm \\
1.58\end{array}$} & $\begin{array}{l}-4.63(-5.58- \\
-3.67)\end{array}$ & \multirow[t]{2}{*}{$\begin{array}{l}5.61 \pm \\
1.20\end{array}$} & $\begin{array}{l}-2.22(-2.96- \\
-1.47\end{array}$ & \multirow[t]{2}{*}{$<0.001$} \\
\hline & & & $<0.001$ & & $<0.001$ & \\
\hline & \multirow[t]{2}{*}{$\begin{array}{l}\text { One month after } \\
\text { the intervention }\end{array}$} & \multirow[t]{2}{*}{$\begin{array}{l}2.12 \pm \\
1.32\end{array}$} & $\begin{array}{l}-5.58(-6.45- \\
-4.72)\end{array}$ & \multirow[t]{2}{*}{$\begin{array}{l}4.22 \pm \\
1.17\end{array}$} & $\begin{array}{l}-3.61(-4.60- \\
-2.62\end{array}$ & \multirow[t]{2}{*}{$\begin{array}{l}< \\
0.001\end{array}$} \\
\hline & & & $<0.001$ & & $<0.001$ & \\
\hline \multirow[t]{5}{*}{ Pain (\%) } & Before & $\begin{array}{l}67.08 \\
\pm 0.26\end{array}$ & Reference & $\begin{array}{l}69.83 \\
\pm 20.88\end{array}$ & Reference & \\
\hline & \multirow[t]{2}{*}{$\begin{array}{l}\text { Immediately after } \\
\text { the intervention }\end{array}$} & \multirow[t]{2}{*}{$\begin{array}{l}41.08 \\
\pm 20.19\end{array}$} & $\begin{array}{l}-26.00 \\
(-31.71- \\
-20.29)\end{array}$ & \multirow[t]{2}{*}{$\begin{array}{l}53.48 \\
\pm 18.60\end{array}$} & $\begin{array}{l}-16.35 \\
(-23.39- \\
-9.31)\end{array}$ & \multirow[t]{2}{*}{0.034} \\
\hline & & & $<0.001$ & & $<0.001$ & \\
\hline & \multirow[t]{2}{*}{$\begin{array}{l}\text { One month after } \\
\text { the intervention }\end{array}$} & \multirow[t]{2}{*}{$\begin{array}{l}24.25 \\
\pm 17.90\end{array}$} & $\begin{array}{l}-42.83 \\
(-49.09- \\
-36.57)\end{array}$ & \multirow[t]{2}{*}{$\begin{array}{l}44.26 \\
\pm 16.24\end{array}$} & $\begin{array}{l}-25.57 \\
(-33.92- \\
-17.21)\end{array}$ & \multirow[t]{2}{*}{$\begin{array}{l}< \\
0.001\end{array}$} \\
\hline & & & $<0.001$ & & $<0.001$ & \\
\hline \multirow[t]{5}{*}{ Disability (\%) } & Before & $\begin{array}{l}62.60 \\
\pm 22.53\end{array}$ & Reference & $\begin{array}{l}65.00 \\
\pm 19.78\end{array}$ & Reference & \\
\hline & \multirow[t]{2}{*}{$\begin{array}{l}\text { Immediately after } \\
\text { the intervention }\end{array}$} & \multirow[t]{2}{*}{$\begin{array}{l}41.35 \\
\pm 19.41\end{array}$} & $\begin{array}{l}-21.25 \\
(-29.11- \\
-13.39)\end{array}$ & \multirow[t]{2}{*}{$\begin{array}{l}54.02 \\
\pm 22.14\end{array}$} & $\begin{array}{l}-10.98 \\
(-17.53- \\
-4.43)\end{array}$ & \multirow[t]{2}{*}{0.042} \\
\hline & & & $<0.001$ & & 0.001 & \\
\hline & \multirow[t]{2}{*}{$\begin{array}{l}\text { One month after } \\
\text { the intervention }\end{array}$} & \multirow[t]{2}{*}{$\begin{array}{l}35.05 \\
\pm 22.83\end{array}$} & $\begin{array}{l}-27.55 \\
(-36.19- \\
-15.94)\end{array}$ & \multirow[t]{2}{*}{$\begin{array}{l}48.42 \\
\pm 20.61\end{array}$} & $\begin{array}{l}-16.58 \\
(-24.48- \\
-8.67)\end{array}$ & \multirow[t]{2}{*}{0.041} \\
\hline & & & $<0.001$ & & $<0.001$ & \\
\hline SPADI (\%) & Before & $\begin{array}{l}64.33 \\
\pm 19.21\end{array}$ & Reference & $\begin{array}{l}66.86 \\
\pm 13.61\end{array}$ & Reference & \\
\hline
\end{tabular}




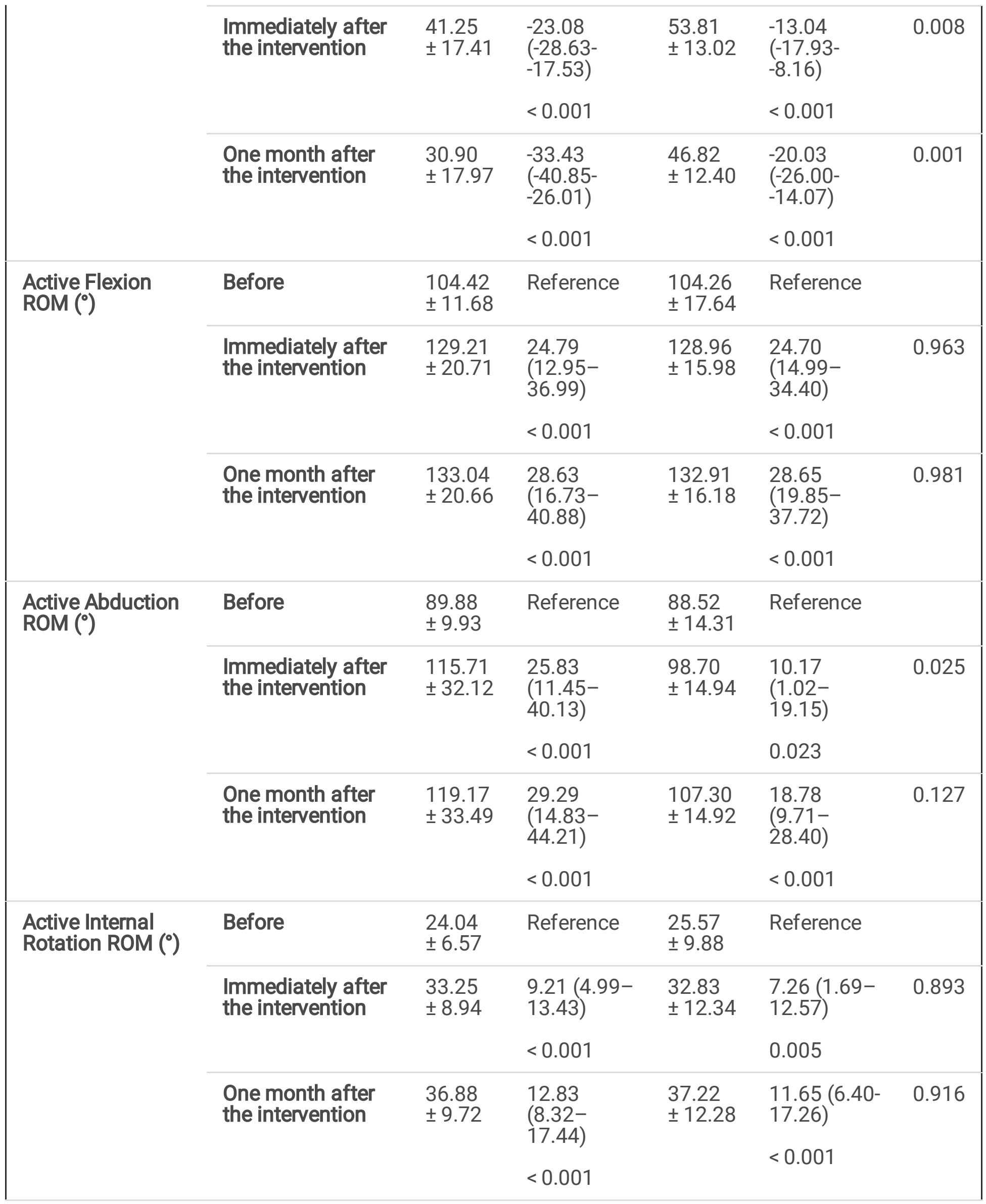

* P-value by repeated-measures statistical test. HBB: Hand behind back; MD: Mean Difference; ROM: Range of motion; SPADI: Shoulder pain and disability index; VAS: Visual Analogue Scale. 


\begin{tabular}{|c|c|c|c|c|c|c|}
\hline \multirow[t]{5}{*}{$\begin{array}{l}\text { Active External } \\
\text { Rotation ROM }\left({ }^{\circ}\right)\end{array}$} & Before & $\begin{array}{l}49.00 \\
\pm 14.19\end{array}$ & Reference & $\begin{array}{l}50.13 \\
\pm 15.33\end{array}$ & Reference & \\
\hline & \multirow[t]{2}{*}{$\begin{array}{l}\text { Immediately after } \\
\text { the intervention }\end{array}$} & \multirow[t]{2}{*}{$\begin{array}{l}59.92 \\
\pm 13.39\end{array}$} & $\begin{array}{l}10.92 \\
(6.72- \\
15.57)\end{array}$ & \multirow[t]{2}{*}{$\begin{array}{l}55.78 \\
\pm 16.31\end{array}$} & $\begin{array}{l}5.65(3.54- \\
7.76)\end{array}$ & \multirow[t]{2}{*}{0.346} \\
\hline & & & $<0.001$ & & $<0.001$ & \\
\hline & \multirow[t]{2}{*}{$\begin{array}{l}\text { One month after } \\
\text { the intervention }\end{array}$} & \multirow[t]{2}{*}{$\begin{array}{l}67.29 \\
\pm 12.99\end{array}$} & $\begin{array}{l}18.29 \\
(12.01- \\
15.57)\end{array}$ & \multirow[t]{2}{*}{$\begin{array}{l}60.13 \\
\pm 16.95\end{array}$} & $\begin{array}{l}10.00 \\
(7.00- \\
13.00)\end{array}$ & \multirow[t]{2}{*}{0.110} \\
\hline & & & $<0.001$ & & $<0.001$ & \\
\hline \multirow[t]{5}{*}{$\begin{array}{l}\text { Passive Flexion } \\
\text { ROM }\left(^{\circ}\right)\end{array}$} & Before & $\begin{array}{l}110.29 \\
\pm 12.69\end{array}$ & Reference & $\begin{array}{l}109.57 \\
\pm 17.01\end{array}$ & Reference & \\
\hline & \multirow[t]{2}{*}{$\begin{array}{l}\text { Immediately after } \\
\text { the intervention }\end{array}$} & \multirow[t]{2}{*}{$\begin{array}{l}132.50 \\
\pm 17.85\end{array}$} & $\begin{array}{l}22.21 \\
(12.18- \\
31.60)\end{array}$ & \multirow[t]{2}{*}{$\begin{array}{l}131.65 \\
\pm 17.03\end{array}$} & $\begin{array}{l}22.09 \\
(11.46- \\
32.72)\end{array}$ & \multirow[t]{2}{*}{0.869} \\
\hline & & & $<0.001$ & & $<0.001$ & \\
\hline & \multirow[t]{2}{*}{$\begin{array}{l}\text { One month after } \\
\text { the intervention }\end{array}$} & \multirow[t]{2}{*}{$\begin{array}{l}137.42 \\
\pm 19.88\end{array}$} & $\begin{array}{l}27.13 \\
(17.32- \\
37.02)\end{array}$ & $\begin{array}{l}136.04 \\
\pm 17.84\end{array}$ & $\begin{array}{l}26.48 \\
(16.43- \\
36.62)\end{array}$ & \multirow[t]{2}{*}{0.805} \\
\hline & & & $<0.001$ & & $<0.001$ & \\
\hline \multirow{5}{*}{$\begin{array}{l}\text { Passive } \\
\text { Abduction ROM } \\
\left({ }^{\circ}\right)\end{array}$} & Before & $\begin{array}{l}92.87 \\
\pm 11.28\end{array}$ & Reference & $\begin{array}{l}91.83 \\
\pm 13.70\end{array}$ & Reference & \\
\hline & \multirow[t]{2}{*}{$\begin{array}{l}\text { Immediately after } \\
\text { the intervention }\end{array}$} & \multirow[t]{2}{*}{$\begin{array}{l}117.21 \\
\pm 31.72\end{array}$} & $\begin{array}{l}24.33 \\
(10.41- \\
38.52)\end{array}$ & $\begin{array}{l}105.96 \\
\pm 21.68\end{array}$ & $\begin{array}{l}14.13 \\
(2.13- \\
25.95)\end{array}$ & \multirow[t]{2}{*}{0.164} \\
\hline & & & 0.001 & & 0.016 & \\
\hline & \multirow[t]{2}{*}{$\begin{array}{l}\text { One month after } \\
\text { the intervention }\end{array}$} & \multirow[t]{2}{*}{$\begin{array}{l}120.12 \\
\pm 35.59\end{array}$} & $\begin{array}{l}27.25 \\
(10.16- \\
43.89)\end{array}$ & $\begin{array}{l}110.70 \\
\pm 21.50\end{array}$ & $\begin{array}{l}18.87 \\
(6.97- \\
30.95)\end{array}$ & \multirow[t]{2}{*}{0.280} \\
\hline & & & 0.001 & & 0.002 & \\
\hline \multirow[t]{3}{*}{$\begin{array}{l}\text { Passive Internal } \\
\text { Rotation ROM }\left(^{\circ}\right)\end{array}$} & Before & $\begin{array}{l}25.42 \\
\pm 7.90\end{array}$ & Reference & $\begin{array}{l}26.39 \\
\pm 11.04\end{array}$ & Reference & \\
\hline & \multirow[t]{2}{*}{$\begin{array}{l}\text { Immediately after } \\
\text { the intervention }\end{array}$} & \multirow[t]{2}{*}{$\begin{array}{l}34.46 \\
\pm 11.46\end{array}$} & $\begin{array}{l}9.04(5.62- \\
12.83)\end{array}$ & \multirow[t]{2}{*}{$\begin{array}{l}35.78 \\
\pm 14.44\end{array}$} & $\begin{array}{l}9.39(3.57- \\
15.03)\end{array}$ & \multirow[t]{2}{*}{0.729} \\
\hline & & & $<0.001$ & & 0.001 & \\
\hline
\end{tabular}

* P-value by repeated-measures statistical test. HBB: Hand behind back; MD: Mean Difference; ROM: Range of motion; SPADI: Shoulder pain and disability index; VAS: Visual Analogue Scale. 


\begin{tabular}{|c|c|c|c|c|c|c|}
\hline & \multirow[t]{2}{*}{$\begin{array}{l}\text { One month after } \\
\text { the intervention }\end{array}$} & \multirow[t]{2}{*}{$\begin{array}{l}39.50 \\
\pm 12.17\end{array}$} & $\begin{array}{l}14.08 \\
(9.78- \\
18.29)\end{array}$ & \multirow[t]{2}{*}{$\begin{array}{l}40.30 \\
\pm 14.75\end{array}$} & $\begin{array}{l}13.91 \\
(7.96- \\
20.14)\end{array}$ & \multirow[t]{2}{*}{0.839} \\
\hline & & & $<0.001$ & & 0.001 & \\
\hline \multirow[t]{5}{*}{$\begin{array}{l}\text { Passive External } \\
\left.\text { Rotation ROM ( }{ }^{\circ}\right)\end{array}$} & Before & $\begin{array}{l}52.17 \\
\pm 16.30\end{array}$ & Reference & $\begin{array}{l}53.65 \\
\pm 15.56\end{array}$ & Reference & \\
\hline & \multirow[t]{2}{*}{$\begin{array}{l}\text { Immediately after } \\
\text { the intervention }\end{array}$} & \multirow[t]{2}{*}{$\begin{array}{l}63.33 \\
\pm 14.58\end{array}$} & $\begin{array}{l}11.17 \\
(3.13- \\
19.02)\end{array}$ & \multirow[t]{2}{*}{$\begin{array}{l}58.00 \\
\pm 15.12\end{array}$} & $\begin{array}{l}4.35(2.19- \\
6.51)\end{array}$ & \multirow[t]{2}{*}{0.235} \\
\hline & & & 0.004 & & & \\
\hline & \multirow[t]{2}{*}{$\begin{array}{l}\text { One month after } \\
\text { the intervention }\end{array}$} & \multirow[t]{2}{*}{$\begin{array}{l}70.79 \\
\pm 11.79\end{array}$} & $\begin{array}{l}18.63 \\
(12.62- \\
24.99)\end{array}$ & $\begin{array}{l}64.87 \\
\pm 15.08\end{array}$ & $\begin{array}{l}11.22 \\
(7.39- \\
14.50)\end{array}$ & \multirow[t]{2}{*}{0.140} \\
\hline & & & $<0.001$ & & $<0.001$ & \\
\hline \multirow[t]{5}{*}{ HBB ROM (cm) } & Before & $\begin{array}{l}-17.05 \\
\pm 9.22\end{array}$ & Reference & $\begin{array}{l}-17.05 \\
\pm 9.22\end{array}$ & Reference & \\
\hline & \multirow[t]{2}{*}{$\begin{array}{l}\text { Immediately after } \\
\text { the intervention }\end{array}$} & \multirow[t]{2}{*}{$\begin{array}{l}-11.63 \\
\pm 7.09\end{array}$} & $\begin{array}{l}5.88(2.58- \\
9.17)\end{array}$ & \multirow[t]{2}{*}{$\begin{array}{l}-13.30 \\
\pm 5.65\end{array}$} & $\begin{array}{l}3.94(0.64- \\
7.23)\end{array}$ & \multirow[t]{2}{*}{0.376} \\
\hline & & & $<0.001$ & & 0.016 & \\
\hline & \multirow[t]{2}{*}{$\begin{array}{l}\text { One month after } \\
\text { the intervention }\end{array}$} & \multirow[t]{2}{*}{$\begin{array}{l}-9.25 \pm \\
5.85\end{array}$} & $\begin{array}{l}8.25(4.07- \\
11.80)\end{array}$ & \multirow[t]{2}{*}{$\begin{array}{l}-10.87 \\
\pm 4.91\end{array}$} & $\begin{array}{l}6.37(2.27- \\
10.02)\end{array}$ & \multirow[t]{2}{*}{0.310} \\
\hline & & & $<0.001$ & & 0.001 & \\
\hline
\end{tabular}

Additionally, the improvement of active abduction ROM in the mobilization group was significantly higher than the control group $(p=0.025)$, however one month after the end of treatment, despite better performance of the mobilization group in improving the active abduction ROM, no statistically significant difference was observed between the two groups $(P=0.127)$. There was no statistically significant difference between the two groups in the improvement of other variables $(P>0.05)$.

\section{Discussion}

In this trial, we compared the pain intensity, functional disability and ROM before and immediately and one month after a) mobilization (in addition to physical therapy and exercises) and b) only physical therapy and exercises as control in participants with FS, and identified some significant differences. There were significant modifications immediately and one month after treatment regarding study outcomes in both groups. However, the differences were significantly higher in mobilization group compared to control group regarding pain intensity and disability, immediately and one month after 
treatment. Patients in mobilization group showed significant higher improvement concerning VAS and SPADI immediately and one month after treatment in comparison with patients in control group. Clinically, physiotherapists have conventionally applied anterior translation of the humeral head so as to improve the external rotation ROM of shoulder. Nevertheless, several investigations reveal that posterior translation is more operative than anterior translation for recovery of external rotation ROM $[46,47]$. So, anterior and posterior joint mobilizations were selected for the present trial with an intensity of Grade I-IV. To our knowledge, this is the first investigation to determine that the one-month outcome of the ACJ mobilization technique in addition to conventional physical therapy and exercises is favorable compared to conventional physical therapy and exercises alone in FS.

The rehabilitation of patients with FS is challenging for patients and also physical therapists. It needs patience along with diverse conventional and surgical interventions in refractory cases. Several treatments, consisting of exercise, massage, and modalities have been encouraged for re-establishment of a pain-free state and ordinary use of the upper limb but any single best treatment intervention has not been recommended. Manual therapy modalities, frequently combined with physical therapy, have been revealed to consequence positively in terms of pain, ROM and function in different studies $[25,41,48]$. It has been established, that joint mobilization techniques can decrease the accompanying glenohumeral rotational deficits, particularly external rotation $[46,49,50]$. Mobilization is characterized by a lowfrequency and small- or large-amplitude movements used to advance the shoulder capsule extensibility and stretch the stiffened soft tissues [51,52]. Joint mobilization methods develop a series of mechanical modifications. Particularly, favorable effects on the joint ROM comprise diminished adhesions, renovation of collagen, and improved fiber sliding [25]. Additional theory correlated to these consequences is the neurophysiological effect acquired throughout joint mobilization methods. This consequence is principally elucidated by the motivation of peripheral mechanoreceptors and the nociceptor dissuasion [25].

Another important finding of this study is that patients underwent mobilization in addition to physical therapy and exercises exhibited significantly higher improvement regarding active abduction ROM in comparison with patients underwent only physical therapy and exercises immediately after treatment, however one month after the end of treatment, no statistically significant difference was observed between the two groups.

Recently, Duzgun et al. [25] compared the efficacy of scapular mobilization, manual posterior capsule stretching, and the combination of these two techniques in the treatment of FS patients. Consistent with our findings, Duzgun established an acute improvement in the ROM of the shoulder joint after mobilization, manual stretching, and the combined application of methods, without any advantage of any method. Maricar et al. [53] has shown that mobilization in combination with exercise therapy for 15 weeks significantly decreased pain intensity and improved ROM in patients with FS. Furthermore, Nicholson [54] has suggested that passive mobilization in combination with active exercises for 4 weeks improved passive abduction compared to exercises alone. However, in another study, both exercise and manual therapy and exercise alone were similarly effective in the management of FS [55]. 
This study has some limitations that should be considered. First, the study applied a single-blinded design. It was impossible to have a double-blind design as the clinician will clearly know what intervention and treatment they are providing. Second, the sample size of study was not large enough to generalize the results to other populaces. Third, the ROM examinations were done with a typical goniometer, which always has a standard measurement error in comparison with more reliable ROM measurement techniques or equipment; however, it is extensively applied in the clinical settings. Forth, as this treatment is additionally applied to the usual physical therapy methods, it is hard to display the isolated properties of mobilization.

In spite of its limitations, the trial's clinical importance is particularly high, as it gives evidence that mobilization in addition to conventional physical therapy and active exercises can provide considerably positive outcomes in improving the pain and disability in comparison with conventional physical therapy and active exercises in patients with FS. Additionally, the ACJ mobilization technique in addition to physical therapy and exercise seems to be more effective than the other two interventions in increasing the active abduction shoulder ROM. These results, if confirmed by upcoming studies, can suggest a new therapeutic method for shoulder disorders and injuries.

\section{Conclusions}

In conclusion, our trial established the positive effects of ACJ mobilization technique on the pain, disability and ROM of the shoulder joint in patients with FS. Nevertheless, additional investigation is required to confirm the effectiveness of mobilization techniques in the treatment of FS.

\section{Abbreviations}

ACJ

Acromioclavicular joint

FS

Frozen shoulder

HBB

hand behind back

HP

Hot bag

NSAIDs

Non-steroidal anti-inflammatory drugs

ROM

range of motion

SPADI

Shoulder Pain and Disability Index

TENS

Transcutaneous Electrical Nerve Stimulation 
VAS

visual analogue scale

\section{Declarations}

\section{Ethics approval and consent to participate}

The investigation has been done according to the principles of the Declaration of Helsinki and Good Clinical Practice. Patients were provided written informed consent before any measurement was done. Patients' personal data about patients were conserved in a database to keep patients' security. The study protocol has been approved by the Ethics Committee of the Research Vice-Chancellor of Tabriz University of Medical Sciences (IR.TBZMED.REC.1397.671). The study was also registered in the clinical trial registry under number IRCT20100605004104N7 code.

\section{Consent for publication}

Not applicable.

\section{Availability of data and materials}

All the necessary data are presented herewith. However if needed, raw data on excel format can be availed on reasonable request from the corresponding author.

\section{Competing interests}

All authors declare that they have no competing interests.

\section{Funding}

Funding was received from the Deputy of Research, Faculty of Medicine, Tabriz University of Medical Sciences, Tabriz, Iran. The funding body had no role in the design of the study, collection, analysis, or interpretation of the data, or writing of the manuscript.

\section{Authors' contributions}

M.R., S.R.K. and N.D. contributed to conception and design of the study, data analysis and interpretation, and drafting the manuscript. S.R.K. collected all data and contributed to data interpretation and drafting the manuscript. S.R.K., B.E., B.I. and N.D. drafted the first manuscript. All authors read and approved the final manuscript.

\section{Acknowledgements}

We are appreciative to all of the participants who helped us to conduct this investigation. The authors would like to acknowledge the staffs of the Physical Medicine and Rehabilitation Research Center of 
Tabriz University of Medical Sciences for their support with this trial. The results provided in this article were a part of a thesis by S. Ranjbar Kiyakalayeh.

\section{Availability of data and materials}

The datasets used and analyzed during the current study are available from the corresponding author on reasonable request.

\section{References}

1. Picavet, H. and J. Hazes, Prevalence of self reported musculoskeletal diseases is high. Annals of the rheumatic diseases, 2003. 62(7): p. 644-650.

2. Fejer, R., K.O. Kyvik, and J. Hartvigsen, The prevalence of neck pain in the world population: a systematic critical review of the literature. European spine journal, 2006. 15(6): p. 834-848.

3. Côté, P., et al., The burden and determinants of neck pain in workers. European Spine Journal, 2008. 17(1): p. 60-74.

4. Hogg-Johnson, S., et al., The burden and determinants of neck pain in the general population. European Spine Journal, 2008. 17(1): p. 39-51.

5. Vázquez Delgado, E., J. Cascos-Romero, and C. Gay Escoda, Myofascial pain syndrome associated with trigger points: a literature review.(I): Epidemiology, clinical treatment and etiopathogeny. Medicina Oral, Patología Oral y Cirugia Bucal, 2009. 14(10): p. 494-498.

6. Fernández-de-las-Peñas, C., et al., Referred pain from myofascial trigger points in head, neck, shoulder, and arm muscles reproduces pain symptoms in blue-collar (manual) and white-collar (office) workers. The Clinical journal of pain, 2012. 28(6): p. 511-518.

7. Treaster, D., et al., Myofascial trigger point development from visual and postural stressors during computer work. Journal of electromyography and kinesiology, 2006. 16(2): p. 115-124.

8. laroshevskyi, O.A., et al., Non-pharmacological treatment of chronic neck-shoulder myofascial pain in patients with forward head posture. Wiadomosci lekarskie (Warsaw, Poland: 1960), 2019. 72(1): p. 84-88.

9. Jafri, M.S., Mechanisms of myofascial pain. International scholarly research notices, 2014. 2014.

10. Jull, G. and M. Sterling, Bring back the biopsychosocial model for neck pain disorders. Manual therapy, 2009. 14(2): p. 117.

11. Ilter, L., et al., Efficacy of pulsed and continuous therapeutic ultrasound in myofascial pain syndrome: a randomized controlled study. American Journal of Physical Medicine \& Rehabilitation, 2015. 94(7): p. 547-554.

12. Suvarnnato, T., et al., The effects of thoracic manipulation versus mobilization for chronic neck pain: a randomized controlled trial pilot study. Journal of physical therapy science, 2013. 25(7): p. 865871. 
13. Barlow, J., A. Turner, and C. Wright, Long-term outcomes of an arthritis self-management programme. British Journal of Rheumatology, 1998. 37(12): p. 1315-1319.

14. Escolar-Reina, P., et al., Self-management of chronic neck and low back pain and relevance of information provided during clinical encounters: an observational study. Archives of physical medicine and rehabilitation, 2009. 90(10): p. 1734-1739.

15. Mohr, A.R., B.C. Long, and C.L. Goad, Effect of foam rolling and static stretching on passive hipflexion range of motion. Journal of sport rehabilitation, 2014. 23(4): p. 296-299.

16. Esenyel, M., N. Caglar, and T. Aldemir, Treatment of myofascial pain. American journal of physical medicine \& rehabilitation, 2000. 79(1): p. 48-52.

17. van der Windt, D.A., et al., Ultrasound therapy for musculoskeletal disorders: a systematic review. Pain, 1999. 81(3): p. 257-271.

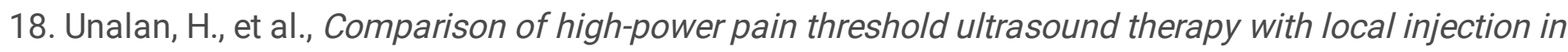
the treatment of active myofascial trigger points of the upper trapezius muscle. Archives of physical medicine and rehabilitation, 2011. 92(4): p. 657-662.

19. Moghtaderi, A., S. Khosrawi, and F. Dehghan, Extracorporeal shock wave therapy of gastroc-soleus trigger points in patients with plantar fasciitis: A randomized, placebo-controlled trial. Advanced biomedical research, 2014. 3.

20. Cho, Y.-S., et al., Effects of the combined treatment of extracorporeal shock wave therapy (ESWT) and stabilization exercises on pain and functions of patients with myofascial pain syndrome. Journal of Physical Therapy Science, 2012. 24(12): p. 1319-1323.

21. Jeon, J.H., et al., The effect of extracorporeal shock wave therapy on myofascial pain syndrome. Annals of rehabilitation medicine, 2012. 36(5): p. 665-674.

22. Shrivastava, S.K. and Kailash, Shock wave treatment in medicine. J Biosci, 2005. 30(2): p. 269-75.

23. Sohn, M.K., et al., Spasticity and electrophysiologic changes after extracorporeal shock wave therapy on gastrocnemius. Annals of rehabilitation medicine, 2011. 35(5): p. 599.

24. Park, K.D., et al., High-versus low-energy extracorporeal shock-wave therapy for myofascial pain syndrome of upper trapezius: A prospective randomized single blinded pilot study. Medicine (Baltimore), 2018. 97(28): p. e11432.

25. Ji, H.M., H.J. Kim, and S.J. Han, Extracorporeal shock wave therapy in myofascial pain syndrome of upper trapezius. Annals of rehabilitation medicine, 2012. 36(5): p. 675.

26. Simons, D., et al., Travell \& Simons' myofascial pain and dysfunction the trigger point manual. 2th ed. 1999, Baltimore: Williams \& Wilkins.

27. Appropriate body-mass index for Asian populations and its implications for policy and intervention strategies. Lancet, 2004. 363(9403): p. 157-63.

28. Lee, P.H., et al., Validity of the International Physical Activity Questionnaire Short Form (IPAQ-SF): a systematic review. Int J Behav Nutr Phys Act, 2011. 8: p. 115. 
29. Baghiani-Moghaddam, M., et al., Comparing the results of pedometer-based data and International Physical Activity Questionnaire (IPAQ). J Health Syst Res, 2013. 9(6): p. 605-12.

30. Vafainajar, A., et al., The Effectiveness of Physical Activity Training on Depersonalization and lack of accomplishment of Employees (Persian). Health Education and Health Promotion 2015. 3(2): p. 116-124.

31. Hoet, J.P. and F.D. Lukens, Carbohydrate metabolism during pregnancy. Diabetes, 1954. 3(1): p. 112.

32. Haefeli, M. and A. Elfering, Pain assessment. Eur Spine J, 2006. 15 Suppl 1: p. S17-24.

33. Lauche, R., et al., Clinically meaningful differences in pain, disability and quality of life for chronic nonspecific neck pain-a reanalysis of 4 randomized controlled trials of cupping therapy. Complementary therapies in medicine, 2013. 21(4): p. 342-347.

34. Abbott, J.H. and J. Schmitt, Minimum important differences for the patient-specific functional scale, 4 region-specific outcome measures, and the numeric pain rating scale. Journal of Orthopaedic \& Sports Physical Therapy, 2014. 44(8): p. 560-564.

35. Sciotti, V.M., et al., Clinical precision of myofascial trigger point location in the trapezius muscle. Pain, 2001. 93(3): p. 259-266.

36. Fischer, A., Documentation of myofascial trigger points. Archives of physical medicine rehabilitation, 1988. 69(4): p. 286-291.

37. Fabio Antonaci, M., Pressure algometry in healthy subjects: inter-examiner variability. Scand J Rehab Med, 1998. 30(3): p. 8.

38. Asiri, F., et al., Effects of patient-specific three-dimensional lumbar traction on pain and functional disability in patients with lumbar intervertebral disc prolapse. Nigerian journal of clinical practice 2020. 23(4): p. 498-502.

39. Vernon, H. and S. Mior, The Neck Disability Index: a study of reliability and validity. Journal of manipulative and physiological therapeutics, 1991.

40. Mousavi, S.J., et al., Translation and validation study of the Iranian versions of the Neck Disability Index and the Neck Pain and Disability Scale. Spine, 2007. 32(26): p. E825-E831.

41. Taheri, P., B. Vahdatpour, and S. Andalib, Comparative study of shock wave therapy and Laser therapy effect in elimination of symptoms among patients with myofascial pain syndrome in upper trapezius. Adv Biomed Res, 2016. 5: p. 138.

42. Pesco, M.S., E. Chosa, and N. Tajima, Comparative study of hands-on therapy with active exercises $v s$ education with active exercises for the management of upper back pain. Journal of manipulative and physiological therapeutics, 2006. 29(3): p. 228-235.

43. Lakens, D., Calculating and reporting effect sizes to facilitate cumulative science: a practical primer for t-tests and ANOVAs. Front Psychol, 2013. 4(863).

44. Shah, J.P., et al., Biochemicals associated with pain and inflammation are elevated in sites near to and remote from active myofascial trigger points. Archives of physical medicine and rehabilitation, 
2008. 89(1): p. 16-23.

45. Gur, A., et al., Comparison of the efficacy of ultrasound and extracorporeal shock wave therapies in patients with myofascial pain syndrome: A randomized controlled study. Journal of musculoskeletal Pain, 2013. 21(3): p. 210-216.

46. Lee, J.-H. and E.-Y. Han, A comparison of the effects of PNF, ESWT, and TPI on pain and function of patients with myofascial pain syndrome. Journal of musculoskeletal Pain, 2013. 25(3): p. 341-344.

47. Király, M., T. Bender, and K. Hodosi, Comparative study of shockwave therapy and low-level laser therapy effects in patients with myofascial pain syndrome of the trapezius. Rheumatology international, 2018. 38(11): p. 2045-2052.

48. Király, M., T. Bender, and K.J.R.i. Hodosi, Comparative study of shockwave therapy and low-level laser therapy effects in patients with myofascial pain syndrome of the trapezius. Rheumatol Int, 2018. 38(11): p. 2045-2052.

49. Han, H., et al., The effects of extracorporeal shock wave therapy on pain, disability, and depression of chronic low back pain patients. Journal of physical therapy science, 2015. 27(2): p. 397-399.

50. Simons, D.G., J.G. Travell, and L.S. Simons, Myofascial pain and dysfunction: upper half of body. 1999: Williams \& Wilkins.

51. Ottomann, C., et al., Prospective randomized trial of accelerated re-epithelization of skin graft donor sites using extracorporeal shock wave therapy. Journal of the American College of Surgeons, 2010. 211(3): p. 361-367.

52. Shah, J.P. and E.A. Gilliams, Uncovering the biochemical milieu of myofascial trigger points using in vivo microdialysis: an application of muscle pain concepts to myofascial pain syndrome. J Bodyw Mov Ther, 2008. 12(4): p. 371-384.

53. Maricar, N., C. Shacklady, and L. McLoughlin, Effect of Maitland mobilization and exercises for the treatment of shoulder adhesive capsulitis: a single-case design. Physiother Theory Pract, 2009. 25(3): p. 203-17.

54. Mangus, B.C., et al., Basic principles of extremity joint mobilization using a Kaltenborn approach. Journal of Sport Rehabilitation, 2002. 11(4): p. 235-250.

55. Ali, S.A. and M. Khan, Comparison for efficacy of general exercises with and without mobilization therapy for the management of adhesive capsulitis of shoulder - An interventional study. Pak J Med Sci, 2015. 31(6): p. 1372-6.

\section{Figures}




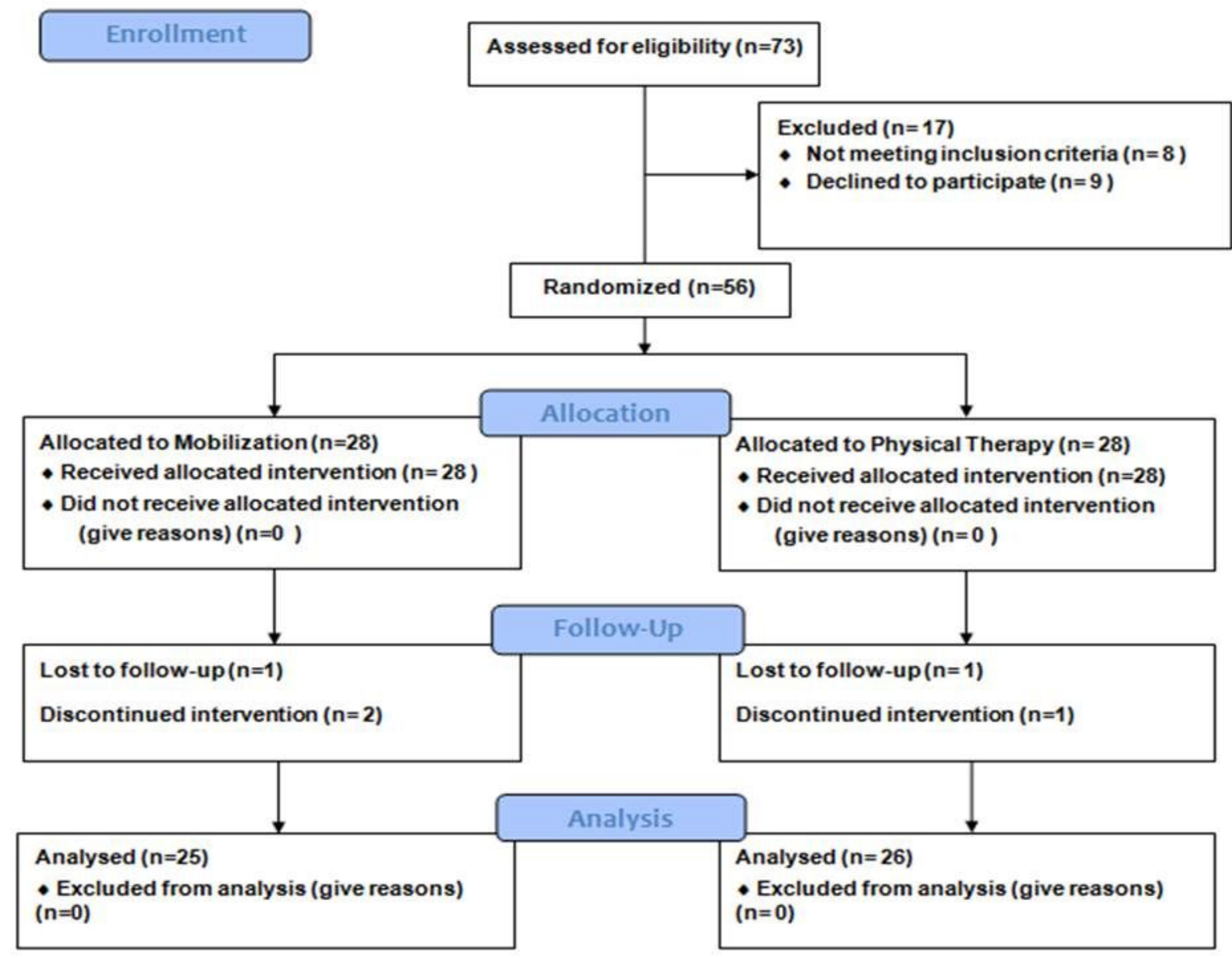

Figure 1

Flowchart of participants 